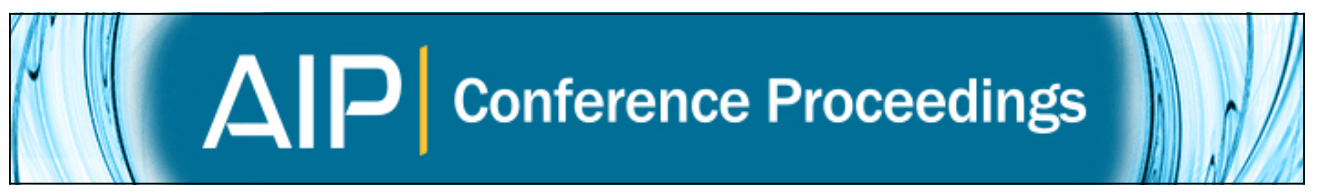

\title{
Matter-matter entanglement for quantum networks
}

Julien Laurat, Kyung Soo Choi, Hui Deng, and H. J. Kimble

Citation: AIP Conference Proceedings 1110, 239 (2009); doi: 10.1063/1.3131316

View online: http://dx.doi.org/10.1063/1.3131316

View Table of Contents:

http://scitation.aip.org/content/aip/proceeding/aipcp/1110?ver=pdfcov

Published by the AIP Publishing

Articles you may be interested in

Pair-wise Entanglement Characterizing a Quantum State of an Atomic Ensemble Storing a Squeezed Light

AIP Conf. Proc. 1110, 265 (2009); 10.1063/1.3131323

Testing Quantum Memories Via Entanglement Verification

AIP Conf. Proc. 1110, 257 (2009); 10.1063/1.3131320

Entanglement and Decoherence in a Microscopic-Macroscopic system AIP Conf. Proc. 1110, 211 (2009); 10.1063/1.3131309

Measurement of Entanglement in Time Domain-an Experiment towards Demonstration of EPR paradox with Continuous Variable using Laser Pulses

AIP Conf. Proc. 1110, 197 (2009); 10.1063/1.3131306

Energy-Time Entangled Qutrits: Bell Tests and Quantum Communication

AIP Conf. Proc. 734, 249 (2004); 10.1063/1.1834427 


\title{
Matter-matter entanglement for quantum networks
}

\author{
Julien Laurat *, Kyung Soo $\mathrm{Choi}^{\dagger}$, Hui Deng ${ }^{\dagger}$ and H. J. Kimble ${ }^{\dagger}$ \\ ${ }^{*}$ Laboratoire Kastler Brossel, Universite P. et M. Curie, Ecole Normale Superieure and CNRS, \\ UPMC, Case 74, 4 place Jussieu, 75252 Paris Cedex 05, France. \\ ${ }^{\dagger}$ Norman Bridge Laboratory of Physics 12-33, California Institute of Technology, \\ Pasadena, California 91125, USA.
}

\begin{abstract}
Developments in quantum information science rely critically on entanglement, as its distribution between different parties enables quantum communication protocols, such as quantum key distribution or teleportation. This talk focused on two different ways to generate heralded entanglement between matter systems, a critical requirement for scalable quantum networking.
\end{abstract}

Keywords: Entanglement, Quantum memory, Quantum repeater PACS: $03.67 . \mathrm{Mn}, 03.65 . \mathrm{Yz}, 03.67 . \mathrm{Hk}, 42.50 . \mathrm{Dv}$

\section{INTRODUCTION}

Quantum control of entanglement between material systems is an essential capability for quantum networks and scalable quantum communication architectures $[1,2]$. In recent years, significant advances have been achieved in the control of the quantum states of atomic systems, including entanglement of trapped ions [3, 4] and between macroscopic quantum spins [5]. By following the seminal paper of Duan, Lukin, Cirac and Zoller $(D L C Z)[6]$, entanglement between single collective excitations stored in two remote atomic ensembles has also been demonstrated [7]. In the $D L C Z$ protocol [6], entanglement is created in a probabilistic but heralded way from quantum interference in the measurement process $[8,9,10]$. The detection of a photon from one or the other atomic ensemble in an indistinguishable fashion results in an entangled state with one collective spin excitation shared coherently between the ensembles. The first section will describe such generation [11].

However, as it will be shown later, an inherent drawback of this approach is the compromise between the amount of entanglement and its preparation probability, leading intrinsically to low count rate for high entanglement. In the second section, we will present a protocol where entanglement between two atomic ensembles is created by coherent mapping of an entangled state of light [12]. By splitting a single-photon and subsequent state transfer, we separate the generation of entanglement and its storage. After a programmable delay, the stored entanglement is mapped back into photonic modes with overall efficiency of $17 \%$. Improvements of single-photon sources [13] together with our protocol will enable "on-demand" entanglement of atomic ensembles.

\footnotetext{
CP1110, Quantum Communication, Measurement and Computing (QCMC),

The Ninth International Conference, edited by A. Lvovsky

(C) 2009 American Institute of Physics 978-0-7354-0647-6/09/\$25.00
} 

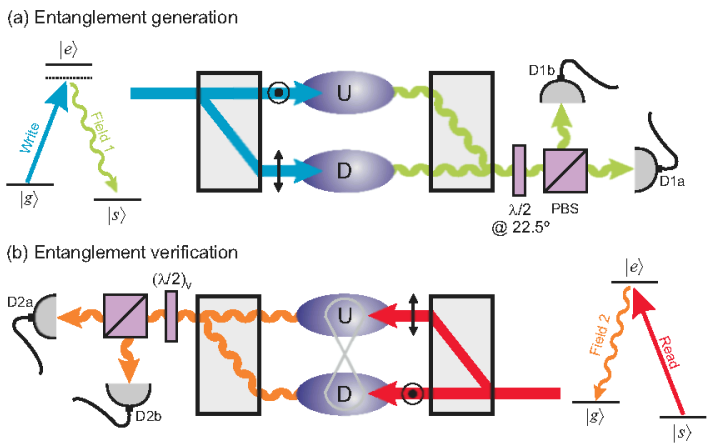

FIGURE 1. (a) A weak write pulse is split into two paths separated by $1 \mathrm{~mm}$ by a birefringent crystal and excites simultaneously two atomic samples, $U, D$. The resulting fields $1_{U, D}$ are combined at the polarizing beamsplitter (PBS) and sent to the single-photon detectors $D_{1 a, 1 b}$. A detection event at $D_{1 a}$ or $D_{1 b}$ heralds the creation of entanglement [7]. (b) After a user-controlled storage time $\tau$, entanglement is verified by mapping the atomic state to propagating light fields $2_{U, D}$ by way of strong read pulses. Tomography of the fields $2_{U, D}$ is then achieved. The atomic cloud is initially prepared in the ground state $|g\rangle .\{|g\rangle,|s\rangle,|e\rangle\}$ denote the Cesium hyperfine levels $\left\{\left|6 S_{1 / 2}, F=4\right\rangle,\left|6 S_{1 / 2}, F=3\right\rangle,\left|6 P_{3 / 2}, F=4\right\rangle\right\}$, respectively. Note that the fields $1_{U, D}$ and $2_{U, D}$ are detected with a small angle relative to the classical beams, which is not represented here for the sake of simplicity.

\section{MEASUREMENT-INDUCED ENTANGLEMENT}

The generation of entanglement following the DLCZ approach is illustrated in Fig. 1. A single cloud of cesium atoms in a magneto-optical trap is used; two ensembles are defined by different optical paths $1 \mathrm{~mm}$ apart. This separation is obtained by the use of birefringent crystals close to the cloud, which separate orthogonal polarizations. At $40 \mathrm{~Hz}$, the trap magnetic field is switched off for $7 \mathrm{~ms}$. After waiting $3 \mathrm{~ms}$ for the magnetic field to decay, the two samples are simultaneously illuminated with $30 \mathrm{~ns}$ long and $10 \mathrm{MHz}$ red-detuned write pulses, at a rate of $1.7 \mathrm{MHz}$. Given the duty cycle of the experiment, the effective rate is $180 \mathrm{kHz}$. Spontaneous Raman scattered fields induced by the write beams are collected into single-mode fibers, defining for each ensemble optical modes that we designate as fields $1_{U, D}$ with $50 \mu \mathrm{m}$ waist and a $3^{\circ}$ angle relative to the direction of the write beams [14]. Fields $1_{U, D}$ are frequency filtered to block spontaneous emission from atomic transitions $|e\rangle \rightarrow|g\rangle$, which do not herald the creation of a collective excitation. After this stage, and before detection, fields $1_{U, D}$ are brought to interfere on a polarizing beam-splitter. A detection event at $D_{1 a, 1 b}$ that arises indistinguishably from either of the fields $1_{U, D}$ projects the atomic ensembles into an entangled state where, in the ideal case, one collective excitation is coherently shared between the $U, D$ ensembles $[6,7]$.

A model-independent determination of entanglement based upon quantum tomography of the fields $2_{U, D}$ obtained by sending a read pulse has been developed in the Supplemental Information accompanying Ref. [7]. The model consists of reconstructing a reduced density matrix, $\tilde{\rho}_{2_{U}, 2_{D}}$, obtained from the full density matrix by restriction to the subspace with no more than one photon per mode. We also assume that all off-diagonal 


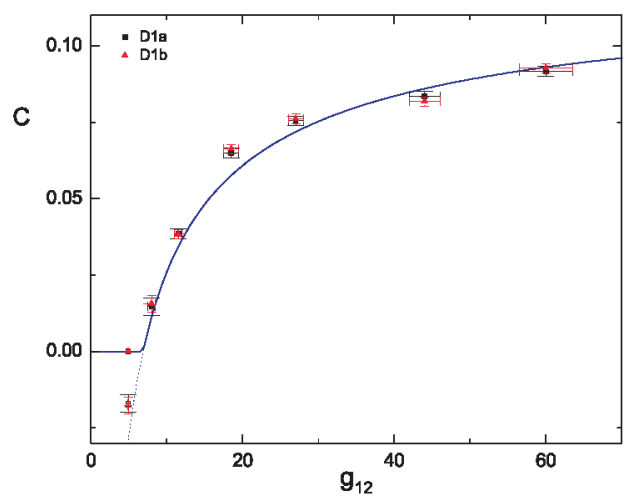

FIGURE 2. Concurrence $C$ as a function of the normalized cross correlation function $g_{12}$, for the two possible heralding events (detection at $D_{1 a}$ or $D_{1 b}$ ).

elements between states with different numbers of photons vanish. The model thus leads to a lower bound for entanglement. As detailed in Ref. [7], $\widetilde{\rho}_{2_{U}, 2_{D}}$ can be written in the photon-number basis $|n\rangle|m\rangle$ with $\{n, m\}=\{0,1\}$ as follows:

$$
\tilde{\rho}_{2_{U}, 2_{D}}=\frac{1}{P}\left(\begin{array}{cccc}
p_{00} & 0 & 0 & 0 \\
0 & p_{01} & d & 0 \\
0 & d^{*} & p_{10} & 0 \\
0 & 0 & 0 & p_{11}
\end{array}\right)
$$

Here, $p_{i j}$ is the probability to find $i$ photons in mode $2 U$ and $j$ in mode $2_{D} ; d$ is the coherence term between the $|1\rangle|0\rangle$ and $|0\rangle|1\rangle$ states; and $P=p_{00}+p_{01}+p_{10}+p_{11}$. From $\tilde{\rho}_{2_{U}, 2_{D}}$, one can calculate the concurrence $C$, which is a convenient monotone measurement of entanglement ranging from 0 for a separable state to 1 for a maximally entangled state:

$$
C=\max \left(0, C_{0}\right) \text { with } C_{0}=\frac{1}{P}\left(2|d|-2 \sqrt{p_{00} p_{11}}\right) .
$$

In the regime of low excitation and detection probabilities in which the current experiment is performed, the vacuum component $p_{00}$ can be approximated by $p_{00} \sim 1-p_{c}$, while the terms $p_{01}$ and $p_{10}$ are given by $p_{10}=p_{01}=p_{c} / 2 . p_{c}$ is the conditional probability of detecting a photon in field 2 from one ensemble following a detection event for field 1 .

To investigate the scaling of entanglement with excitation probability $\chi$, we determined $C$ for various values of $\chi$ for fixed memory time $\tau=200 \mathrm{~ns}$ (Figure 2). As $\chi$ increases, higher order terms cannot be neglected, precisely as in parametric down conversion. A convenient parameter to assess the excitation regime of each ensemble is the normalized intensity cross correlation function $g_{12}$ between field 1 and field 2 [14], defined as $g_{12}=p_{12} /\left(p_{1} p_{2}\right)$ with $p_{12}$ the joint probability for detection events from field 1 and 2 in a given trial and $p_{i}$ the probability for unconditional detections in field $i$. In 
the ideal case, this function is related to the excitation probability $\chi$ by $g_{12}=1+1 / \chi$, where $g_{12}>2$ defines the nonclassical border in the ideal case and $g_{12} \gg 2$ being the single-excitation regime for the ensembles.

\section{MAPPING PHOTONIC ENTANGLEMENT INTO AND OUT}

The drawback of the previous measurement induced protocol is that entanglement and count rate are inherently correlated. Here, we demonstrate the reversible mapping of an entangled state into a quantum memory. The mapping is obtained by using adiabatic passage based upon dynamic Electromagnetically Induced Transparency (EIT) [15, 16]. Storage and retrieval of optical pulses have been demonstrated previously, for both classical pulses [17], and for single photon pulses [18, 19]. Adiabatic transfer of a collective excitation has been demonstrated between two ensembles coupled by a cavity mode [20], which can provide a suitable approach for generating on-demand entanglement over short distances.

In our experiment, entanglement between two atomic ensembles $L_{a}, R_{a}$ is created by first splitting a single photon into two modes $L_{i n}, R_{i n}$ to generate an entangled state of light. This entangled field state is then coherently mapped to an entangled matter state for $L_{a}, R_{a}$. On demand, the stored atomic entanglement for $L_{a}, R_{a}$ is reversibly converted back into entangled photonic modes $L_{\text {out }}, R_{\text {out }}$. As opposed to the original $D L C Z$ scheme, our approach is inherently deterministic, suffering principally from the finite efficiency with which single excitations can be mapped to and from an atomic memory, with efficiency $\simeq 50 \%$ having been achieved. Moreover, the contamination of entanglement for the $L_{a}, R_{a}$ ensembles from processes involving 2 excitations can be arbitrarily suppressed (independent of the mapping probabilities) with continuing advances in on-demand single photon sources [13]. Our experiment thereby provides a promising avenue to distribute and store entanglement deterministically over remote atomic ensembles for quantum networks.

The experimental setup is depicted in Fig. 3. Our single photon source is based on Raman transitions in an optically thick cesium ensemble $[6,14]$. This system generates $28 \mathrm{~ns}$-long single photons (resonant with the $6 S_{1 / 2}, F=4 \leftrightarrow 6 P_{3 / 2}, F^{\prime}=4$ transition) in a heralded fashion [14]. The single photons are polarized at $45^{\circ}$ from the eigenpolarizations of the beam displacer $B D_{1}$ (Fig. 1b), which splits them into entangled optical modes $L_{i n}, R_{i n}$ (called the signal modes) to produce, in the ideal case, the state in the following form :

$$
\frac{1}{\sqrt{2}}\left(\left|0_{L_{i n}}\right\rangle\left|1_{R_{\text {in }}}\right\rangle+e^{i \phi_{r e l}}\left|1_{L_{i n}}\right\rangle\left|0_{R_{i n}}\right\rangle\right) .
$$

The next stage consists in coherently mapping photonic entanglement for $L_{i n}, R_{i n}$ into atomic ensembles $L_{a}, R_{a}$ (called the memory ensembles) within a single cloud of cold cesium atoms in a magneto-optical trap (MOT) (Fig. 1c). Ensembles $L_{a}, R_{a}$ are defined by the well-separated optical paths of the entangled photonic modes $L_{i n}, R_{i n}$. To avoid dissipative absorption for the fields in modes $L_{i n}, R_{i n}$ for our choice of polarization [18], we initially spin-polarize the atomic ensemble into $\left|F=4, m_{F}=0\right\rangle$. A synchronous clock governs the trials of both the single photon source and memory 


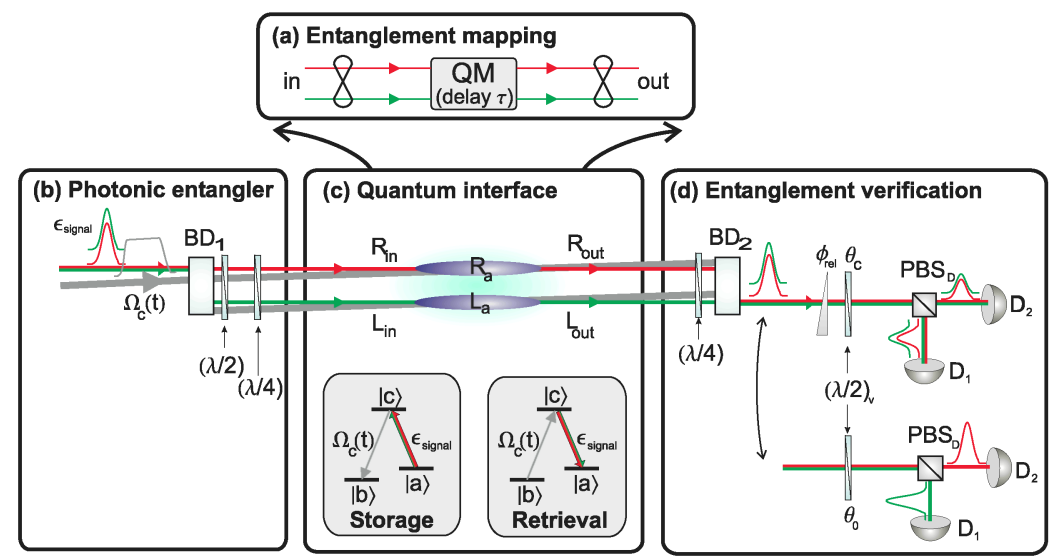

FIGURE 3. (a) Mapping of an entangled state of light into and out of a quantum memory (QM) with storage time $\tau$. (b) Photonic "entangler": A beam displacer $B D_{1}$ splits an input single photon into two orthogonally polarized, entangled modes $L_{i n}, R_{i n}$, which are spatially separated by $1 \mathrm{~mm}$. With waveplates $\lambda / 2$ and $\lambda / 4$, the signal fields $\varepsilon_{\text {signal }}$ for $L_{i n}, R_{\text {in }}$ and control fields $\Omega_{c}^{(L, R)}(t)$ are transformed to circular polarizations with the same helicity along each path $L, R$, and copropagate with angle of $3^{\circ}$. (c) Photonic entanglement between $L_{i n}, R_{i n}$ is coherently mapped into the memory ensembles $L_{a}, R_{a}$ by switching $\Omega_{c}^{(L, R)}(t)$ off adiabatically. After a programmable storage time, the atomic entanglement is reversibly mapped back into optical modes $L_{\text {out }}, R_{\text {out }}$ by switching $\Omega_{c}^{(L, R)}(t)$ on. Relevant energy diagrams for the storage and retrieval processes are shown in the insets. States $|a\rangle,|b\rangle$ are the hyperfine ground states $F=4$, $F=3$ of $6 S_{1 / 2}$ in atomic cesium; state $|c\rangle$ is the hyperfine level $F^{\prime}=4$ of the state $6 P_{3 / 2}$. (d) Verification : After a $\lambda / 4$ plate, the beam displacer combines modes $L_{\text {out }}, R_{\text {out }}$ into one beam with orthogonal polarizations. With $(\lambda / 2)_{v}$ at $\theta_{c}=22.5^{\circ}$ before the polarization beamsplitter $\left(\mathrm{PBS}_{D}\right)$, single photon interference is recorded at detectors $D_{1}, D_{2}$ by varying the relative phase $\phi_{\text {rel }}$ by a Berek compensator. With $(\lambda / 2)_{v}$ at $\theta_{0}=0^{\circ}$, photon statistics for each mode $L_{\text {out }}, R_{\text {out }}$ are measured independently.

ensembles with a period of $575 \mathrm{~ns}$. Initially, the strong control fields $\Omega_{c}^{(L, R)}$ (resonant with the $6 S_{1 / 2}, F=3 \leftrightarrow 6 P_{3 / 2}, F^{\prime}=4$ transition) open transparency windows $\Omega_{c}^{(L, R)}(0)$ in $L_{a}, R_{a}$ for the signal modes. As the wavepacket of the signal field propagates through each ensemble, the control fields $\Omega_{c}^{(L, R)}(t)$ are turned off in 20 ns by an electro-optical intensity modulator, thus coherently transforming the fields of the respective signal modes to collective atomic excitations within $L_{a}, R_{a}$. This mapping leads to heralded entanglement between ensembles $L_{a}, R_{a}$. After a user-defined delay, chosen here to be $1.1 \mu \mathrm{s}$, the atomic entanglement is converted back into entangled photonic modes by switching on the control fields $\Omega_{c}^{(L, R)}(t)$. Figure 4 gives the entaglement measurement before and after storage. A transfer efficiency, defined as the ratio of input and output concurrence, of $20 \%$ is demonstrated.

In conclusion, we have reported two different ways of generating heralded entanglement between atomic ensembles. Our second protocol alleviates the significant drawback of probabilistic protocols [6], where low preparation probabilities prevent its potential scalability. Our strategy can be incorporated into recent extensions of [6] for efficient scaling for high fidelity quantum communication. 


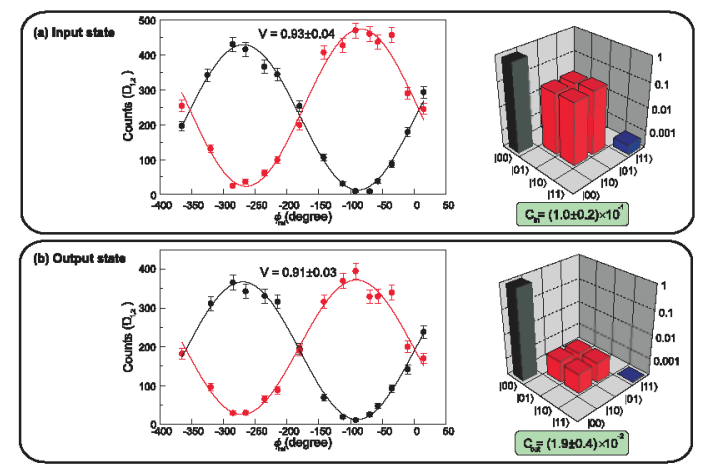

FIGURE 4. Entanglement for the input (a) and output (b) optical modes. The figure shows the interference fringes and the reconstructed density matrices (in $\log$ scale) for the photonic modes a, at the input of the memory and $\mathbf{b}$, at the output after storage and retrieval. The estimated concurrence is given in each case. Each point of the fringe is taken for $20,000(100,000)$ heralding events for the input (output) state.

\section{ACKNOWLEDGMENTS}

We acknowledge our ongoing collaboration with S. J. van Enk and discussions with D. Felinto, H. de Riedmatten and C.W. Chou. This research is supported by the DTO and by the NSF. J. L. acknowledges financial support from the EU (Marie Curie fellowship) and H. D. from the CPI at Caltech.

\section{REFERENCES}

1. P. Zoller et al., Eur. Phys. J. D 36, 203 (2005).

2. H.J. Briegel et al., Phys. Rev. Lett. 81, 5932 (1998).

3. H. Häffner et al., Appl. Phys. B 81, 151 (2005).

4. C. Langer et al., Phys. Rev. Lett. 95, 060502 (2005).

5. B. Julsgaard, A. Kozhekin, E.S. Polzik, Nature 413, 400 (2001).

6. L.M. Duan et al., Nature 414, 413 (2001).

7. C.W. Chou et al., Nature 438, 828 (2005).

8. R.H. Dicke, Am. J. Phys. 49, 925 (1981).

9. C. Cabrillo et al., Phys. Rev. A 59, 1025 (1999).

10. S. Bose et al., Phys. Rev. A 83, 5158 (1999).

11. J. Laurat, K.S. Choi, H. Deng, C.W. Chou, H.J. Kimble, Phys. Rev. Lett. 99, 180504 (2007).

12. K.S. Choi, J. Laurat, H. Deng, H.J. Kimble, Nature 452, 67 (2008).

13. B. Lounis, M. Orrit, Rep. Prog. Phys. 68, 1129-1179 (2005).

14. J. Laurat, J. et al., Opt. Express 14, 6912-6918 (2006).

15. S.E. Harris, Phys. Today 50, 36-40 (1997).

16. M. Fleischhauer, M.D. Lukin, Phys. Rev. Lett. 84, 5094-5097 (2000).

17. L.V. Hau, S.E. Harris, Z. Dutton, C.H. Behroozi, Nature 397, 594-598 (1999).

18. T.Chaneliére, et al., Nature 438, 833-836 (2005).

19. M.D. Eisaman,et al., Nature 438, 837-841 (2005).

20. J. Simon, H. Tanji, S. Ghosh, V. Vuletić, Nature Physics 3, 765-769 (2007); advance online publication, 23 September 2007 (DOI 10.1038/nphys726). 